\title{
Support of the hyperbolic connectance hypothesis by qualitative stability of model food webs
}

\author{
X. Chen ${ }^{1}$ and J. E. Cohen ${ }^{1,2}$ \\ ${ }^{1}$ Laboratory of Populations, Rockefeller University and Columbia University, 1230 York Avenue, Box 20, \\ New York, NY 10021-6399, USA. Fax:1-212-327-7974, E-mail: cohen@rockefeller.edu \\ ${ }^{2}$ To whom correspondence should be addressed.
}

Key words: Local asymptotic stability; Lotka-Volterra cascade model; Qualitative global asymptotic stability.

\begin{abstract}
Local stability analysis of dynamical models of interacting populations predicted that food web connectance $(C)$ is proportional to $1 / S$ where $S$ is species richness. This "hyperbolic connectance hypothesis" was initially supported by analyses of documented food webs. This study shows that the qualitative global asymptotic stability of the Lotka-Volterra cascade model with a finite number of species predicts a relationship between connectance and species richness that agrees closely with the hyperbolic connectance hypothesis predicted from the analysis of local asymptotic stability. Moreover, the threshold of the qualitative global asymptotic stability in the Lotka-Volterra cascade model separates food webs in constant environments from those in fluctuating environments. The obvious discrepancy between the $C-S$ relationship based on some recent data and that predicted by the dynamical models could be due to the selection of data.
\end{abstract}

Abbreviations: LVCM - Lotka-Volterra Cascade Model, LAS - Local Asymptotic Stability, QGAS - Qualitative Global Asymptotic Stability.

\section{Introduction}

Food web connectance is the fraction of potential links that are actually engaged (Gardner and Ashby 1970). The connectance is one of the key structural properties of food webs. Recently, the relationship between connectance and species richness has become a controversial issue (Martinez 1992, Hall and Raffaelli 1993, Warren 1994, Havens 1992, 1997, Bersier et al. 1999, Martinez et al. 1999).

The conventional theory of food web connectance is the hyperbolic connectance hypothesis. This hypothesis emerged from the theoretical investigations of the relationship between stability and complexity in ecological and other systems (Gardner and Ashby 1970, May 1972, Pimm 1982). Supposing a randomly assembled food web has $S$ species, connectance $C$ and an average interaction strength $a$, May suggested that, for large $S$, the system would almost certainly be locally asymptotically stable if and only if $S C a^{2}<1$. Assuming a constant $a$ independent of $S$, this equation suggests that, for local stability, at the boundary of the inequality, connectance should be in- versely proportional to species richness, that is, $C \sim 1 / S$. Cohen and Newman $(1984,1985 a)$ showed that $S C a^{2}<1$ is not necessary and sufficient to assure a hyperbolic decline in connectance.

The local asymptotic stability (LAS) analysis of randomly assembled model food webs has been questioned for various reasons as a theoretical paradigm for predicting food web patterns. First, randomly assembled food webs may allow biologically unrealistic structures such as the absence of autotrophs (DeAngelis 1975, Lawlor 1978, Pimm 1984). Second, local stability may not be a good measure of community persistence. May (1974) thought that qualitative stability deserves study as a determinant of the relation between complexity and stability.

Recently, Cohen et al. (1990b) incorporated into the Lotka-Volterra model biologically plausible topological features of the cascade model (Cohen et al. 1990a) to define the Lotka-Volterra cascade model (LVCM). Cohen et al. (1990b) showed that the qualitative global asymptotic stability (QGAS) of the LVCM in the limit as $S \rightarrow \infty$ (extremely large webs) gives a hyperbolic $C-S$ relationship. QGAS goes beyond LAS in two important ways: it is 
"qualitative" because stability depends only on the sign pattern, not the numerical values, of the coefficients of the dynamical equations; and it is "global" because the system returns to its equilibrium following perturbations of any size, not merely following sufficiently small perturbations. The analysis of Cohen et al. (1990b) provides no information about the $C-S$ relationship in webs with a finite number of species.

The prediction of a hyperbolic $C-S$ relationship received support from analyses of some catalogues of food webs (Rejmanek and Stary 1979, Pimm 1982, Briand 1983, Cohen and Newman 1985b, Schoenly et al. 1991, Havens 1997). Early analyses yielded approximately $\mathrm{C} \approx$ $4 S^{-1}$ (Cohen and Newman 1988). This pattern is equivalent to a roughly linear increase of the number of links with species richness, $L \approx 2 S$ (Cohen and Briand 1984, Cohen and Newman 1985b, 1988, Schoenly et al. 1991).

Most early food web data on which these analyses were based are usually side products of surveys made for other purposes. Because of this, the validity of food web properties based on these data was questioned (Paine 1988, Lawton 1989, Winemiller 1989, Martinez 1991, 1992, Hall and Raffaelli 1993). Based on existing and new data, Martinez (1992) proposed the "constant connectance" hypothesis, namely, that the connectance is independent of the number of species $L / S^{2}=k$ ( $k$ is a positive constant). Between the hyperbolic connectance hypothesis and the constant connectance hypothesis, an intermediate hypothesis states that the number of links increases roughly as the $4 / 3$ or $3 / 2$ power of $S$ (Cohen et al. 1986, Schoener 1989, Havens 1992). These alternative $C$ $S$ patterns apparently differ from predictions of dynamical models.

In this study, we show that the QGAS of the LVCM with a finite number of species predicts a hyperbolic $C-S$ relationship which is in striking agreement with the empirical regularity $C=4 S^{-1}$ emerging from the previous catalogues of food webs. The LAS and QGAS of LVCM with a finite number of species provide qualitatively consistent predictions about the $C-S$ relationship. These predictions appear to be consistent with the data when the possible effects of selection of data are considered.

\section{The Lotka-Volterra cascade model (LVCM) and methods of analyses}

The Lotka-Volterra cascade model (LVCM) follows the classic Lotka-Volterra system of ordinary differential equations (May 1974, Pimm 1982, Cohen and Newman 1988, Cohen et al. 1990b). Let $x_{i}$ be the abundance of species $i, e_{i}$ the intrinsic rate of change, $p_{i j}$ the coefficient of interaction of species $j$ with species $i$, and

$\dot{x}_{i}=x_{i}\left(e_{i}+\sum_{j} p_{i j} x_{j}\right)$. The LVCM assumes that the sign patterns of the community matrix, $P=\left(p_{i j}\right){ }_{i, j}$, are defined by the cascade model.

The cascade model has a single parameter $c>0$ which is independent of the number of species $S$. The cascade model assumes that for each pair of species $i, j=1, \ldots, S$ with $i<j$, species $i$ can never eat species $j$, while species $j$ can eat species $i$ with a probability of $c / S(c / S=C$ is the connectance, $0<c \leq S$ ) independently for each pair of species. For large $S$, the parameter $c$ may be interpreted as (nearly) the average number of species eaten by the topranked species, species $S$. Here we suppose all trophic links to be consumer-victim links, which is a special case of the LVCM. If $p_{i j}$ is the per-capita effect of species $j$ on the per-capita rate of growth of species $i$, then we have $\operatorname{Pr}\left(p_{i j}<0 \& p_{j i}>0\right)=c / S$. Here $\operatorname{Pr}($.$) means "probability of$ the event (.)". All the species are assumed to be self-limited, i.e., $\operatorname{Pr}\left(p_{i i}<0\right)$ for all $i$ (Cohen et al. 1990b). We do not assume that every trophic link has dynamical effects, so the graphs of dynamical interactions in our model food webs do not necessarily have to be connected graphs.

If $L$ is the number of undirected non-cannibalistic links, connectance $C$ refers to undirected connectance, and $E($.$) is the mean or expected value, then C=E(L) /[S(S$ 1)/2]. In Martinez's constant connectance hypothesis, the connectance is directed connectance with cannibalism, i.e., $C^{6}=E\left(L^{\varsigma}\right) / S^{2}\left(L^{\prime}\right.$ is the number of directed links, including cannibalistic links). Each undirected consumervictim link counted in $L$ makes two directed links counted in $L^{\prime}$ so that $E\left(L^{\circ}\right)=2 E(L)$.

Each randomly assembled LVCM food web has QGAS, according to conditions given by Logofet (1993), if the digraph of the LVCM food web has no $k$-cycles for $k \geq 3$. (Other conditions given by Logofet are automatically satisfied here.) A randomly assembled LVCM food web has LAS if and only if all the eigenvalues of its Jacobian matrix have a negative real part, i.e., $\operatorname{Re}\left(\lambda_{i}\right)<0$ for all $i$.

Numerical simulations to estimate the probabilities of QGAS and LAS are conducted on randomly constructed LVCM food webs with given $S$ and $C$. The value of $S$ is incremented from 2 to 60 with a step of 2 . The value of $C$ is incremented from 0 to 1 with a step of 0.05 . For each combination of $C$ and $S$ values, a random community matrix $P=\left(p_{i j}\right)_{i, j}$ is generated. First, a uniformly distributed random number $\psi$ is drawn from the interval $(0,1)$ to determine whether to assign a link between the species $i$ and species $j(i<j)$ independently for all pairs of $i, j$. If $\psi \leq C$, then a link is assigned, i.e., $p_{i j}<0$ and $p_{j i}>0$. Then the $p_{i j}$ 
and $p_{i i}$ are each assigned a uniformly distributed random value in the interval $(-1,0)$, and $p_{j i}$ is assigned a uniformly distributed random value in the interval $(0,1)$. All uniform variables are selected independently.

For each combination of values of $C$ and $S, 10000$ such random systems are assembled and the relative frequency $f$ of QGAS or LAS is measured. The upper bound of the standard deviation $S d$ of each estimated probability

$f$ is $S d=\sqrt{f(1-f) / 10000} \leq \sqrt{0.5 \cdot 0.5 / 10000}=0.005$. To correct measures of correlation for the number of fitted parameters, we used corrected $R^{2}=1-\left(1-R^{2}\right)(n-1) /(n-p$ $-1)$, where $n$ is the number of data points, $p$ the number of parameters, and $R^{2}$ the ordinary linear correlation.

\section{Results}

C-S relationship as constrained by dynamical stability

The surfaces in Fig. 1A and 1B show the relationship between $S$ and $C$ at different levels of probability of QGAS and LAS, respectively. As the values of $S$ and $C$ increase, a gradual transition from non-zero probability to zero probability occurs.

The level contours of the probability surfaces of QGAS and LAS, plotted as functions of $C$ and $S$, depict the relationship between $C$ and $S$ at specific levels of probability of QGAS (Fig. 2A) or LAS (Fig. 2B). The top contour in each of Fig. 2A and Fig. 2B, at the probability of stability of 0.0001 , represents the frontier of stability: it separates the region of positive probability of stability
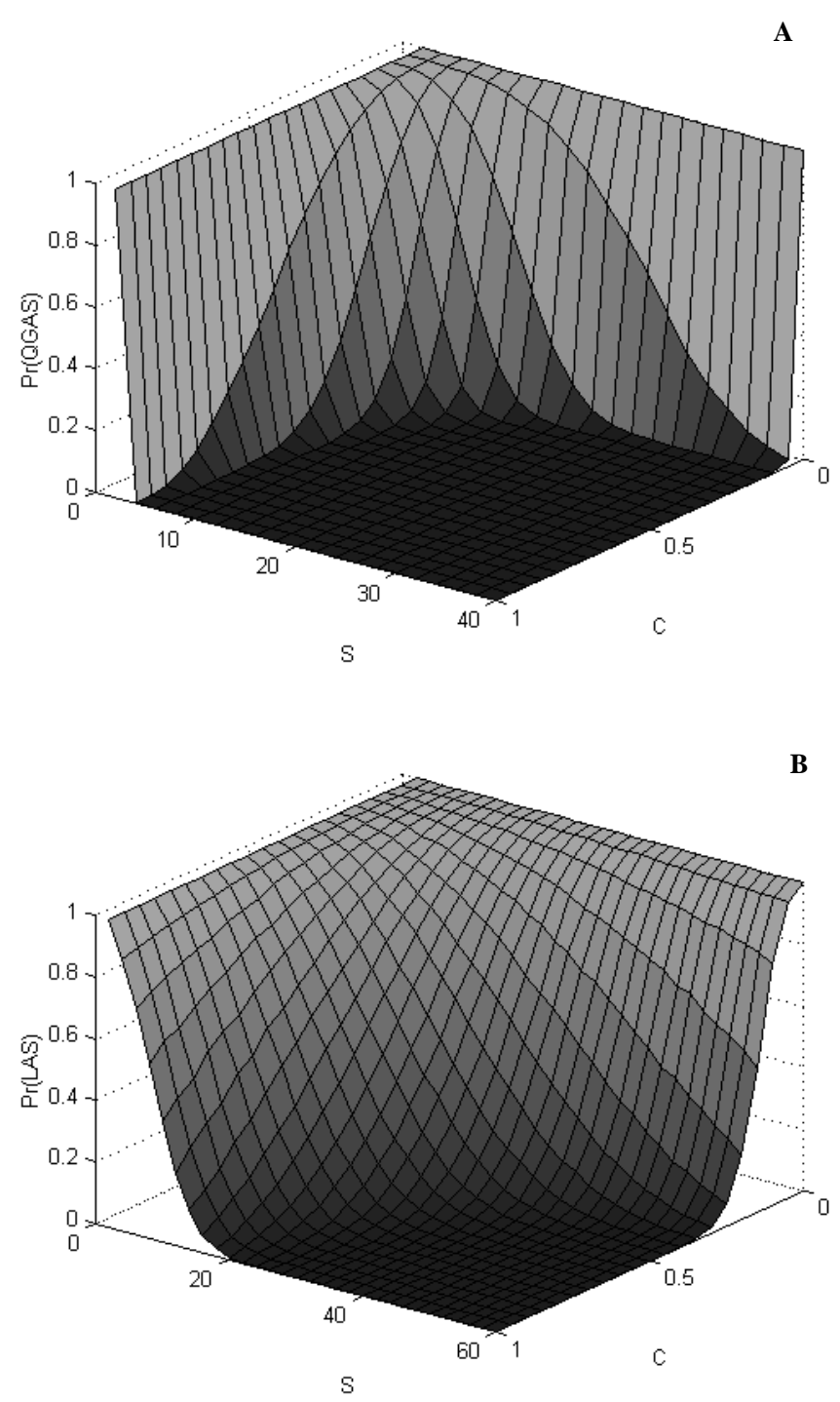

Figure 1. Perspective views of (A) the probability of qualitative global asymptotic stability and (B) the probability of local asymptotic stability in the Lotka-Volterra cascade model as a function of species richness $(S)$ and connectance $(C)$. 
Figure 2. Contours of (A) probabilities of qualitative global asymptotic stability and (B) probabilities of local asymptotic stability in the Lotka-Volterra cascade model as a function of species richness $(S)$ and connectance $(C)$. Each $\bullet$ represents the result of 10,000 simulated webs. The value on each contour curve is the probability at which the contour is taken. Small values of $S$ and $C$ assure a high probability of stability.

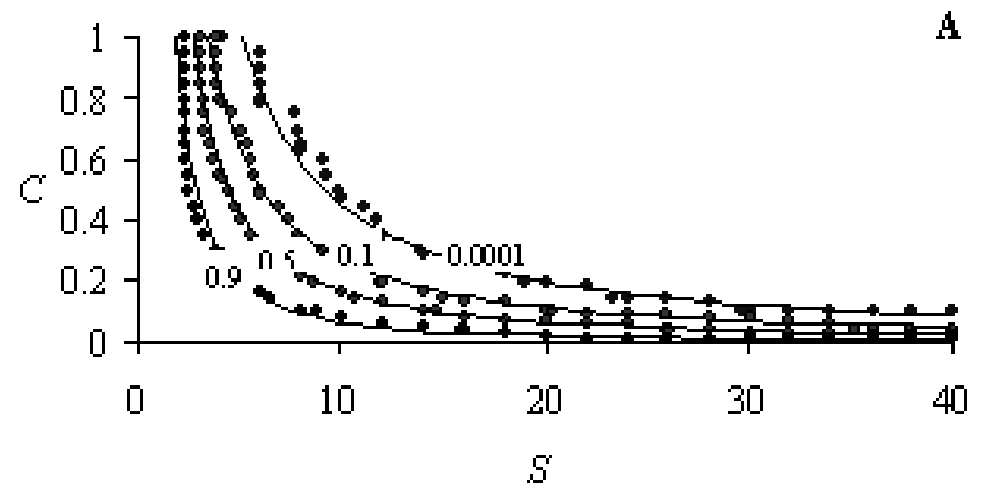

A

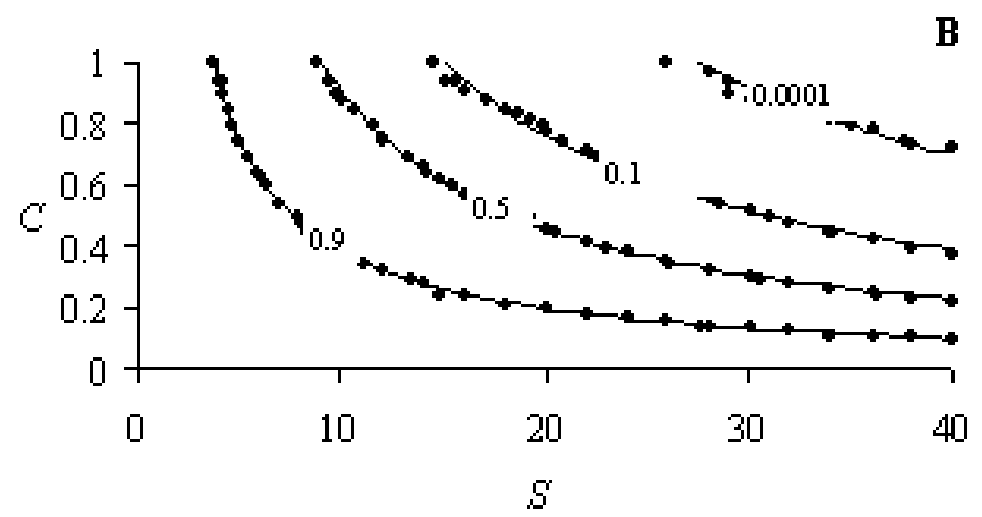

from the region of effectively zero probability of stability. For finite $S$, it is possible that the probability of stability may never be 0 .

The simulated data points at the frontier of QGAS are fitted to the hyperbolic model of $C$ as a function of $S$ with a nonlinear least-squares algorithm, which gives $C=$ $4.5834 S^{-0.9895}$ (corrected $R^{2}=0.9504$ ) (Fig. 3A). The standard deviations of the multiplier and the exponent of $S$ are 0.3729 and 0.0407 , respectively. The hyperbolic form of this $C-S$ relationship is qualitatively insensitive to the probability (here 0.0001 ) chosen to represent the frontier of stability. The fitted $C-S$ curve is very close to that given by the hyperbolic connectance hypothesis $C=4 S^{-1}$ (Cohen and Briand 1984) (Fig. 3A). When the number of links $L$ is plotted against $S$ ( $C$ is converted to $L$ by $L=$ $C S(S-1) / 2)$, the least-squares fit of the simulated data points at the frontier of QGAS is a power function: $L=$ $1.6021 S^{1.0511}$ (corrected $R^{2}=0.9544$ ) (Fig. 3B). The standard deviations of the multiplier and the exponent of $S$ are 0.2711 and 0.0484 , respectively. The fitted $L-S$ plot appears very close to that given by the species-linkage scaling hypothesis $L=2 S$, but clearly departs from that given by the constant connectance hypothesis $\mathrm{L}^{\prime}=0.11 \mathrm{~S}^{2}$ (Fig. 3B) (where the coefficient 0.11 is the median value of the range 0.08-0.14 given by Martinez (1992)) and that given by the intermediate hypothesis $L=0.6713 S^{1.36}$ (Cohen et al. 1986).

At the frontier of LAS (Fig. 4), the $C-S$ and $L-S$ curves fitted by least squares to the simulated data points give $C$ $=19.9999 S^{0.9080}$ (corrected $R^{2}=0.9799$ ) and $L=$ $12.7711 S^{1.0195}$ (corrected $R^{2}=0.9805$ ). The standard deviations of the exponents of $S$ are 0.0257 and 0.0298 in the $C$ - and $L$-equations, respectively. Quantitatively, these curves represent a much more relaxed constraint on food web complexity than do the curves at the frontier of QGAS because, for local stability, a larger number of links is compatible with a given number of species. However, the exponents of the $C$-equation and $L$-equation are close to -1 and 1 respectively, showing that the curves at the frontier of LAS are qualitatively in line with the hyperbolic connectance law. By simple trial-and-error, we found that the fitted $C-S$ and $L-S$ curves at the probabilities of LAS of 0.9 , namely, $C=3.5267 S^{-0.9604}$ (corrected $R^{2}$ $=0.9992$ ) and $L=1.5848 S^{1.0609}$ (corrected $R^{2}=0.9980$ ), are visually very close to the curves given by $C=4 S^{-1}$ and $L=2 S$ (Fig. 4). The standard deviations of the two parameters in the $C$-equation are 0.0239 and 0.0038 , and 

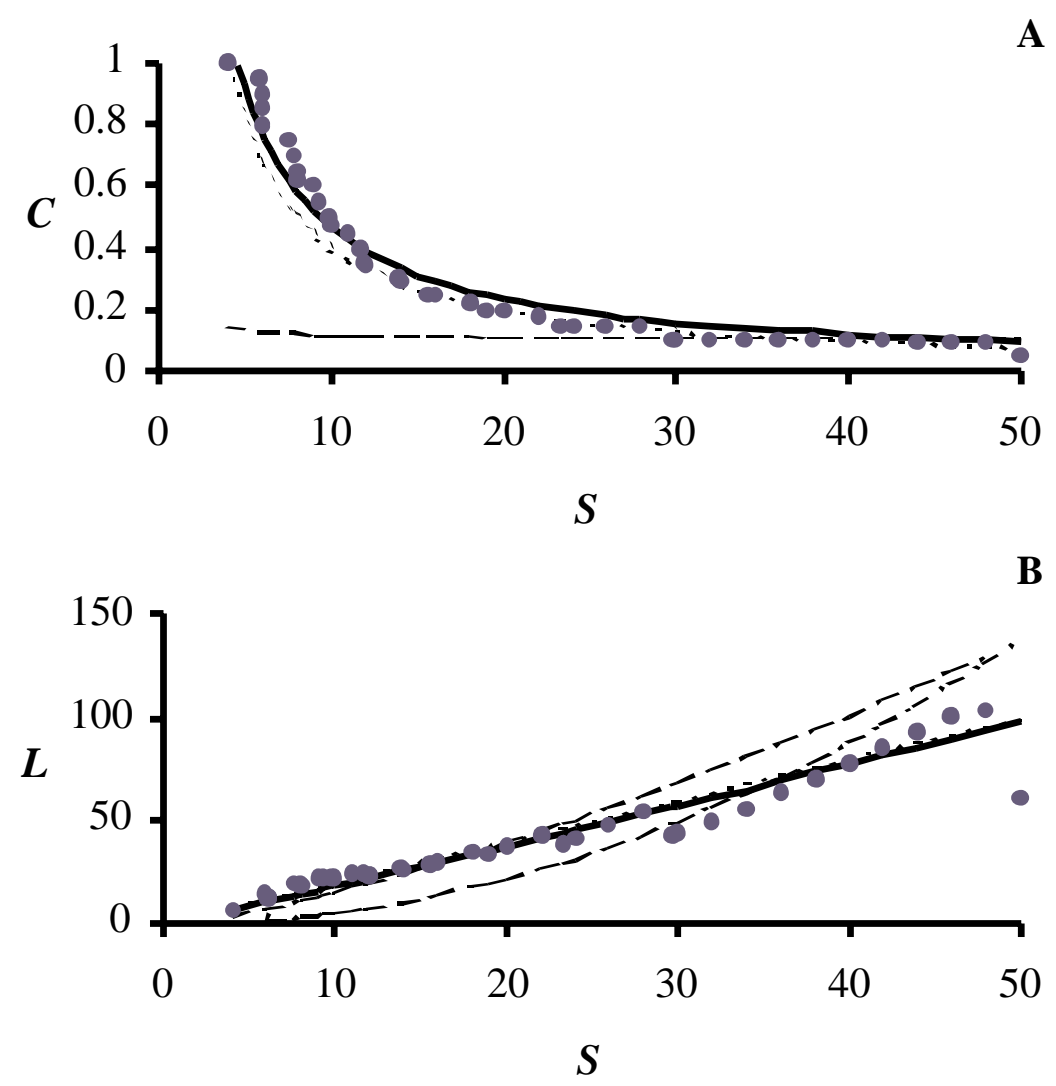

Figure 3. Connectance $(C)$ and number of links $(L)$ in relation to species richness $(S)$ at the frontier (probability of 0.0001 ) of qualitative global asymptotic stability of the Lotka-Volterra cascade model. The curvilinear least-squares fit (--) of the simulated data points $(\bullet)$ gives $C$ $=4.5834 S^{0.9895}, R^{2}=0.9504$ in $\mathrm{A}$, and $L=1.6021 S^{1.0511}, R^{2}=0.9544$ in B. The dotted curves (. . .) are given by the hyperbolic connectance $\left(C=4 S^{-1} ; \mathrm{A}\right)$ and species-link scaling hypotheses $(L=2 S$; B) ; it overlaps the solid curve in $B$. The dashed curves (-.--) are given by the constant connectance hypothesis $L^{\prime} / \mathrm{S}^{2}=0.11$. The broken line (- - ) in $\mathrm{B}$ is given by the intermediate hypothesis $L=0.6713 S^{1.36}$.
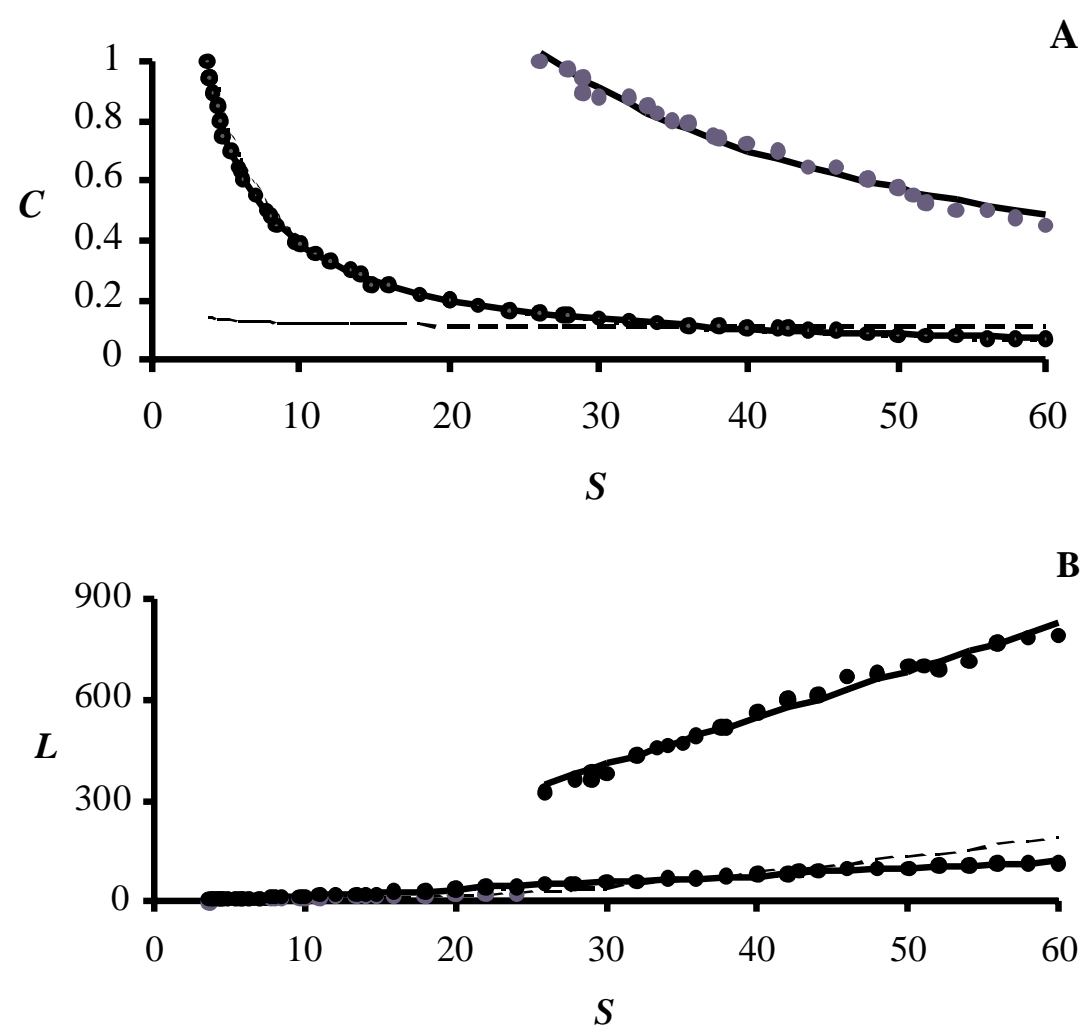

A Figure 4. Connectance $(C)$ and number of links $(L)$ in relation to species richness $(S)$ at the frontier (probability of 0.0001 ) of local asymptotic stability of the LotkaVolterra cascade model (thick line). The least-squares fitted contours at the probability of 0.9 (thin line) are visually very close to the curves given by the hyperbolic connectance $\left(C=4 S^{-1} ; \mathrm{A}\right)$ and species-link scaling $(S=2 L$; B) hypotheses $(-)$. The dotted curve overlaps the solid curve in A. Each dashed curve (---)

B is given by the constant connectance hypothesis. In comparing the hyperbolic connectance hypothesis (where connectance is measured by C) with the constant connectance hypothesis (where connectance is measured by $C^{\prime}$ ), $C^{\prime}$ is converted to $C$ by $C=C^{\prime} S /(S-1)$, so $C$ is not a constant function of $\mathrm{S}$ in the constant connectance hypothesis. 


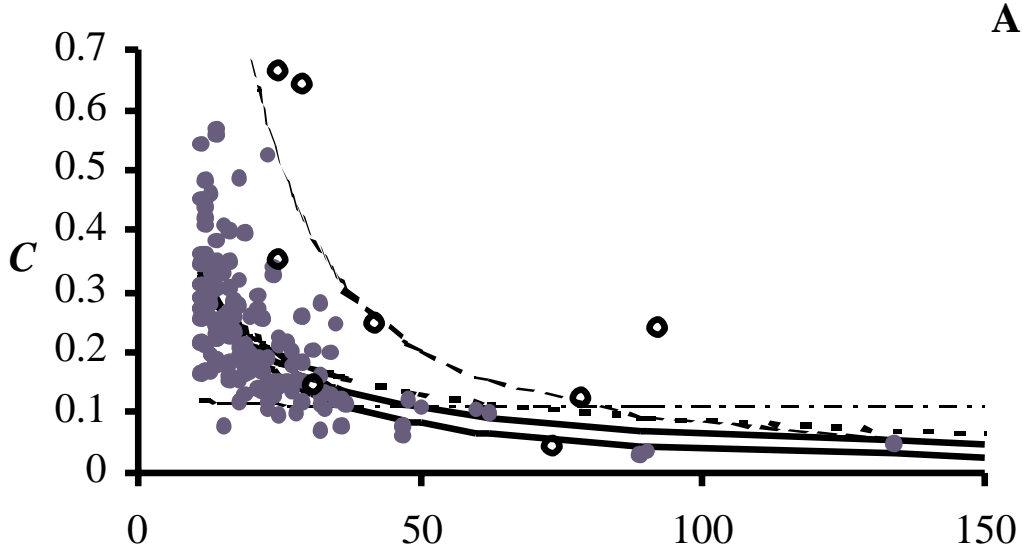

$S$

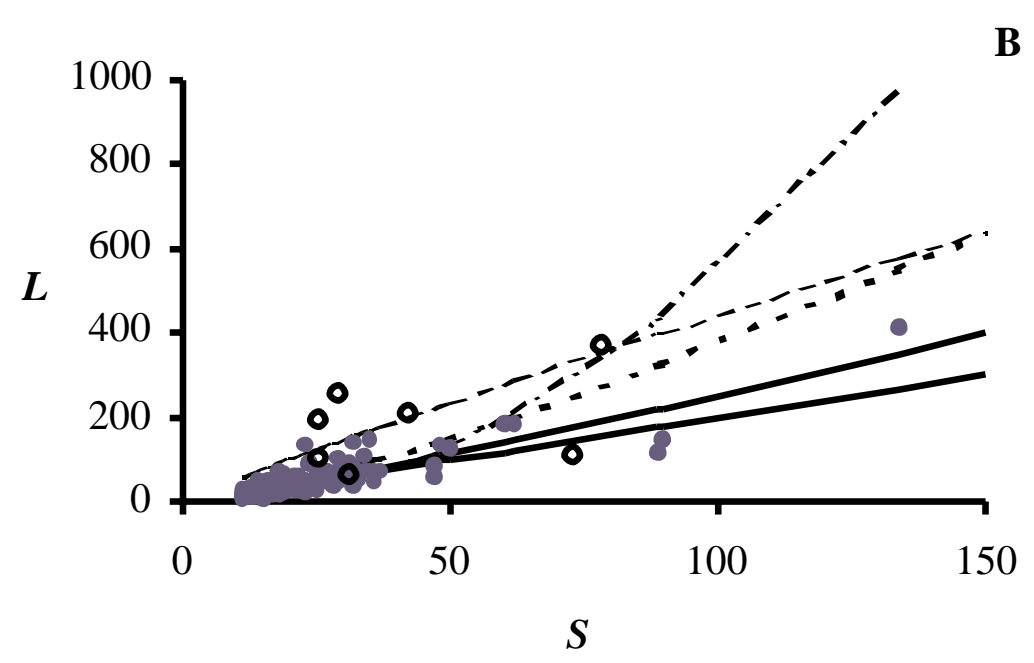

A Figure 5. The empirical relationships between connectance (C) and trophic species richness $(S)$, and between number of links $(L)$ and trophic species richness $(S)$. The solid circles $(\bullet)$ represent 142 webs from the ECOWeB collection, and the open circles (o) represent 8 new and large webs. The best fits of the 142 webs are represented by thick solid lines; the best fits of the total 150 webs are shown by thick dashed lines - - -; the best fits of the 8 new webs are shown by - - - . They are compared with the hyperbolic connectance or species-link scaling hypothesis __ and the constant connectance hypothesis -..-. those in the $L$-equation are 0.0623 and 0.0105 , respectively.

These results indicate that the hyperbolic connectance hypothesis and the mathematically equivalent specieslink scaling hypothesis hold for webs with topological features of the cascade model at the frontiers of LAS and QGAS.

\section{Reconciliation with empirical data}

We evaluate the fitted $C-S$ and $L-S$ equations at the frontier of QGAS using 150 webs with more than 10 trophic species. (A trophic species consists of all organisms identified as having the same sets of prey and the same sets of predators. A trophic species may contain multiple biological species, or may contain a single life stage of a biological species.) This set of 150 webs includes 142 sink or community webs having more than 10 (trophic) species from the 213 webs gathered by Cohen, and 8 new and large webs [1 web from Martinez et al. (1999) and 7 webs from Williams and Martinez (2000)].

For the 142 webs, the curvilinear least-squares regressions give $C=1.930 S^{-0.736}$ (corrected $\left.R^{2}=0.400\right)$ an $L=$ $1.348 S^{1.136}$ (corrected $R^{2}=0.774$ ). The standard deviations of the exponents of S are 0.088 and 0.040 in the two equations, respectively. The two exponents are closer to -1 and 1 , as in the hyperbolic connectance law, than to 0 and 2, as in the constant connectance law. The predictions by the fitted equations appear rather close to that given by the threshold of QGAS and the hyperbolic connectance (link-species scaling) hypothesis, but differ qualitatively from that given by the constant connectance hypothesis (Fig. 5).

For the 8 large webs, curvilinear least-squares regression gives $C=34.373 S^{-1.307}$ (corrected $R^{2}=0.511$ ). The standard deviation of the exponent of $S$ is 0.780 . Neither the hyperbolic connectance hypothesis nor the constant 
connectance hypothesis provides a satisfactory curvilinear least-square fit to $L-S$ relationship in the 8 large webs. Linear least-squares regression of $\log L$ against $\log S$ gives $L=6.708 S^{0.911}$ (corrected $R^{2}=0.346$ ). The standard deviation of the exponent of $S$ is 0.514 . Neither regression is statistically instructive because of the small $R^{2} \mathrm{~s}$ and large standard deviations of the exponents of $S$, but the fitted curves appear visually closer to hyperbolic connectance than to constant connectance (Fig. 5).

Curvilinear least-squares regressions for the 150 webs give $C=1.381 S^{0.605}$ (corrected $R^{2}=0.273$ ) and $L=$ $0.875 S^{1.317}$ (corrected $R^{2}=0.506$ ). The standard deviations of the exponents of $S$ are 0.093 and 0.090 in the two equations, respectively. The fitted $L-S$ curve for this data set is similar to that previously given by Cohen et al. (1986), i.e., $L=0.6713 S^{1.36}$, but the overall fit is quite poor. The curvature and position of the $C-S$ data points resemble the hyperbolic connectance model more than they resemble the constant connectance hypothesis (Fig. 5). The new data do not appear to support the constant connectance hypothesis.
Connectance-species-richness relationship and habitat variability

Food webs in fluctuating environment are less connected (Briand 1983) and have shorter food chains (Briand and Cohen 1987) than those in constant environments. Here we extend the analysis of connectance to all 71 documented food webs with more than 10 trophic species and identified habitat variability compiled by Cohen et al. (1990a). These webs were classified into two categories: 51 webs were labeled as exposed to "fluctuating" environments and 20 webs as exposed to "constant" environments (Cohen et al. 1990a).

In these webs, the average number of trophic species in constant and fluctuating environments are $21.78 \pm 3.60$ and $21.45 \pm 3.96$ (95\% confidence interval), respectively. They are not significantly different from each other (Student's $t$ test, $P=0.9002$ ). However, the connectances in constant and fluctuating environments are $0.33 \pm 0.08$ and $0.20 \pm 0.02$ ( $95 \%$ confidence interval), respectively. The former is significantly higher than the latter (Student's $t$
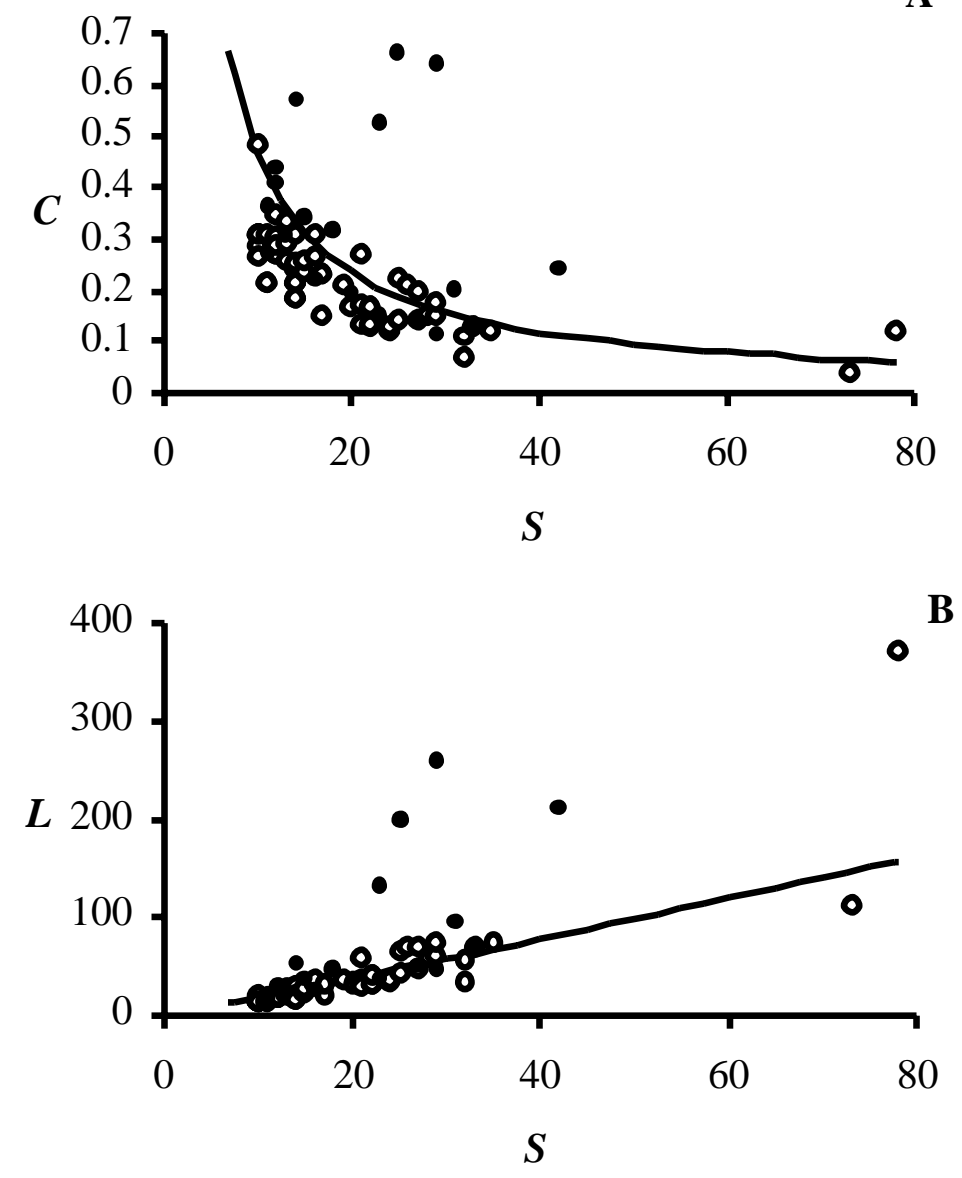

Figure 6. Empirical connectance $(C)$ and empirical number of links $(L)$ of food webs as a function of trophic species richness $(S)$ in fluctuating (o) and constant $(\bullet)$ environments. The connectance and number of links are significantly greater in constant than in fluctuating environments $(P=0.0017)$. The curves are the frontier of qualitative global asymptotic stability (with probability 0.0001 ) of the simulated LotkaVolterra cascade model. 
test, $P<0.0017$ ) (Fig. 6). This finding confirms the results of Briand (1983) based on the analysis of 40 webs (32 webs that have more than 10 trophic species in his analysis are included in this study). Briand (1983) assumed a link of interference competition between any pair of predators that shared at least one common prey, while we do not. Species that share a prey need not necessarily have dynamically significant interference competition.

Of the 51 webs in fluctuating habitats, 43 (84\%) webs have connectance below the frontier of QGAS. In contrast, of the 20 webs in constant habitats, only $9(45 \%)$ webs have connectance below the frontier of QGAS (Fig. 6). The proportion of webs within the frontier of QGAS in fluctuating environments is significantly higher than that in constant environments $(P<0.0093)$.

\section{Discussion}

Cohen et al. (1990b) showed mathematically that a hyperbolic $C-S$ relationship holds at the frontier of QGAS of the LVCM in the limit when $S$ approaches infinity. If all interactions are consumer-victim links, then at the frontier of QGAS,

$$
\lim _{S \rightarrow \infty} C S=1
$$

Our numerical simulations show that the $C-S$ relationship at the frontier of QGAS of the LVCM with a finite number of species is in striking agreement with the empirical regularity $C S=4$. These investigations represent the only effort, suggested by May (1974), to explore the relation of qualitative stability to complexity. The $C-S$ relationship emerging from the QGAS of the LVCM sheds new light on the hyperbolic connectance hypothesis predicted by the LAS (May 1972, Pimm 1982, Auerbach 1984, Cohen and Newman 1984, 1985a, 1988). The QGAS depends only on the topological properties of food webs, that is, only on the sign pattern of the interaction matrix, but is independent of the interaction strengths and initial conditions. Thus, the $C-S$ relationship emerging from the QGAS represents a fundamental structural property of food webs.

The frontier of QGAS separates webs in constant environments from those in fluctuating environments. A food web having QGAS will remain globally stable unless the trophic structure changes. Food webs in fluctuating habitats usually have high variability in the interaction coefficients and intrinsic rates of increase or decrease, as well as frequently perturbed state variables. In fluctuating habitats, persistence of food webs tends to require foodweb structures that produce QGAS. On the other hand, food webs in relatively constant environments usually have lower variability in interaction coefficients and intrinsic rates of increase or decrease, as well as less frequently perturbed state variables. In constant environments, food webs can persist within looser constraints of stability, such as local stability.

The QGAS of the LVCM shows that the hyperbolic connectance hypothesis is supported by formal models and concepts independent of those from which the hypothesis originally derived. The cascade model's predictions of many biologically reasonable properties (although some debates exist) were based on values of a free parameter in the hyperbolic connectance hypothesis $C=$ $4 S^{-1}$ or something similar. When the cascade model is embedded into the Lotka-Volterra dynamical model, qualitative stability predicts an equivalent hyperbolic $C-S$ relationship, independently of any prior assumptions about connectance.

The $C-S$ relationship predicted by the QGAS of the LVCM agrees well with the empirical regularity emerging from the early food web catalogues which have been considered to be of poor quality, but differs considerably from the relationship derived from the new and large webs. Moreover, alternative hypotheses were derived based on different sets of documented food webs (Cohen et al. 1986, Schoener 1989, Warren 1990, 1994, Martinez 1991, Havens 1992). How should one view the disparity among alternative hypotheses and between the theoretical prediction and the patterns from the new large webs? Here we first argue that the concept of a link in the dynamic models need not be compatible with that in empirical food-web graphs. Then we suggest that the prediction of hyperbolic connectance from dynamical stability is not necessarily in contradiction with different patterns if the underlying assumptions are relaxed or altered.

\section{A trophic link in dynamic models has a different meaning from a link in empirical food-web graphs}

In the empirical webs, a link is drawn if one species eats another species. In the dynamical models, a link is assigned only if the trophic or other (e.g., behavioral) interaction has dynamical effects on predator or prey or both species. In food-web studies, investigators usually try to detect and report all possible feeding links, based on observations, stomach analyses, or sometimes guesses based on knowledge of biology of the species (Paine 1988, Martinez 1991, Polis 1991, Warren 1994). Some but not all of these links may be dynamically effective. In real ecosystems, some feeding links may have no or trivial dynamical consequence (Paine 1980, 1988, Lawton 1989) or only a one-way dynamical consequence, such as the do- 
nor-controlled and recipient-controlled interactions (Pimm 1980, Lawton 1989, Cohen et al. 1990b). Links having no or trivial dynamical effects are omitted in dynamical models. The $C-S$ relationship predicted by the dynamical models might differ from that emerging from the new large webs because of the difference in the meaning of a trophic link. The food web catalogues from which the empirical hyperbolic connectance pattern was derived were usually side-products of surveys oriented toward studies of ecosystem functioning or population dynamics, not detailed food web studies. These webs may have grasped only the essential and dominant trophic groups and links of the systems and may have ignored the less significant interactions. In comparison with detailed webs that include all trophic links regardless of their dynamic significance, the earlier simplified webs may have links that are more in line with the assumptions of dynamical models.

In dynamical models, a link represents a locally and instantaneously effective trophic interaction, while in empirical food-web graphs, the species and interactions are usually lumped over time and space. In reality, many species shift diets seasonally or with age or ontogenetic stages (Price 1984, Paine 1988, Schoenly and Cohen 1991). Diets may also change as a result of optimal foraging if there are changes in the relative abundance of alternative food resources (Price 1984, Krebs and Davies 1993). Real trophic links also vary in space (Paine 1980, 1988, Gloss and Lake 1994) but reported food-web graphs often combine data from several study sites. At each particular time and local site, the number of actual links in a food web is less than that in the reported cumulative web. The old food web catalogues usually focused on the trophic groups and links that exist over broad spatial and temporal scales, and thus might be more in line with dynamical models than detailed webs which include all temporary and local links.

Experiments suggest that real food webs may be characterized by a few strong links and many weak links (Paine 1980, 1988, 1992, Lawton 1992). Those weak links have, by definition, insignificant dynamical consequences and thus may often have been ignored in food webs documented with low sampling effort. Our analysis shows that the connectance in the food webs documented with relatively low sampling effort is more compatible with the connectance constrained by the qualitative global stability of the dynamical models than is the connectance in the food webs documented with high sampling effort. This finding suggests that only some reported trophic links have dynamical consequences relevant to global qualitative stability.
Various relations between connectance (involving trophic links) and web sizes have been proposed (Schoener 1989, Cohen et al. 1990a, Warren 1990, 1994, Martinez 1992, Havens 1992) based on different sets of data. These empirical $C-S$ patterns are sensitive to the selection of data sets. Most of these empirical patterns lack theoretical justifications.

The hyperbolic connectance hypothesis (supposing $C$ $\left.\approx L / S^{2}\right)$ implies a constant link density $(L / S=k)$ across food webs of different sizes. The constant connectance hypothesis implies a link density that is directly proportional to $S(L / S=k S)$. An intermediate hypothesis states that the link density increases roughly according to $L / S=$ $k S^{1 / 2}$ (Schoener 1989). More food web data need to be collected and compiled with standardized procedures to test these hypotheses further (Cohen et al. 1993).

Hyperbolic connectance is not the only pattern that constraints of dynamical stability could imply

Dynamical stability implies a hyperbolic $C-S$ relation under certain assumptions or conditions. Changing any assumptions or conditions may alter the $C-S$ relationship. An examination of these assumptions and conditions may explain alternative patterns of connectance.

1) One assumption is that the magnitude and distribution of interaction coefficients and the evenness of equilibrium populations are scale-independent (that is, independent of the number of species). If the interaction strengths and population evenness are scale-dependent, patterns of connectance other than the hyperbolic connectance may be produced. If $\mathrm{CSa}^{2}<1$, which is May's (1972) condition, $C$ may remain constant or increase with $S$ if the average interaction strength $a$ decreases with increasing $S$. So far, no evidence indicates that interaction strengths and population evenness are scale-invariant.

2) Another implicit assumption underlying the hyperbolic connectance relation is that the species richness $S$ of food webs is not related to environmental variability. However, species diversity of many taxa decreases with increasing environmental variability (MacArthur 1976). The species richness of food webs could be positively correlated with environmental constancy on ecological time scales (though not necessarily on evolutionary time scales). Previous (Briand 1983, Briand and Cohen 1987) and our current analyses of the existing catalogues of food webs suggested that food webs have a greater connectance in constant environments than in fluctuating environments. If the variation in $S$ is largely due to the environmental variability, then the variation in connectance is also related to the environmental variability, rather than 
simply responding to $S$. In this case, it would not be surprising if link density increased with $S$.

3) The hyperbolic connectance represents the $C-S$ relationship at a specific probability of stability. There has been no justification to explain why all observed food webs should evolve to a threshold of stability associated with the same probability. Many observed communities may be undergoing succession. Food webs in transient communities under succession usually have lower complexity than food webs in mature climax communities (Price 1984). These transient communities usually have not reached the complexity that the threshold of stability would allow. It is not impossible that, as a transient community evolves, both the number of species and connectance may increase at the same time. If different food webs in a collection are at different stages of succession, a positive correlation between link density and web size may be produced.

4) The conventional theory of food web dynamics assumes that the complexity of food webs is constrained by asymptotic or long-term stability. When food webs are subjected to environmental fluctuations, their transient dynamical responses may affect their persistence more than their asymptotic stability. Food-web persistence in the transient phase may deviate from the long-term asymptotic stability in fluctuating environments. The ability to persist in the transient phases under fluctuating environments may at least partly explain why $C$ and $S$ sometimes follow other than the hyperbolic relationship.

The connectance may be determined not only by the intrinsic biological mechanisms and dynamical stability, but also by successional stage and by the features of the environments such as productivity, and frequency and amplitude of perturbations. Connectance is determined by many system-specific factors in addition to the species richness. The $C-S$ relationship derived from real food webs is sensitive to the collection of webs. Large data sets are required to compare webs from different types of habitats.

Acknowledgements: We thank three anonymous referees for valuable comments. This work was supported in part by U.S. National Science Foundation grant BSR9207293 and DEB9981552. J.E.C. acknowledges with thanks the hospitality of Mr. and Mrs. William T. Golden during this work.

\section{References}

Auerbach, M. J. 1984 Stability, probability and the topology of food webs. In: D. R. Strong, Jr. D. Simberloff, L. G. Abele and A. B. Thistle (eds), Ecological Communities: Conceptual Issues and Evidence. Princeton University Press. pp. 412-436.
Bersier, L.-F., P. Dixon and G. Sugihara. 1999. Scale-invariant behavior of the link density property in food webs: a matter of sampling effort? Amer. Nat. 153: 676-682.

Briand, F. 1983. Environmental control of food web structure. Ecology 64: 253-263.

Briand, F. and J. E. Cohen. 1987. Environmental correlates of food chain length. Science 238: 956-960.

Cohen, J.E., R.A. Beaver, S.H. Cousins, D.L. DeAngelis, L. Goldwasser, K.L. Heong, R.D. Holt, A.J. Kohn, J.H. Lawton, N. Martinez, R.O. O’Malley, L.M. Page, B.C. Patten, S.L. Pimm, G.A. Polis, M. Rejmanek, T.W. Schoener, K. Schoenly, W.G. Sprules, J.M. Teal, R.E. Ulanowicz, P.H. Warren, H.M. Wilbur, P. Yodzis. 1993. Improving food webs. Ecology 74: 252-259.

Cohen J. E. and F. Briand. 1984. Trophic links of community food webs. Proceedings of the Royal Society of London B 224: 421448.

Cohen, J. E., F. Briand and C. M. Newman. 1986. A stochastic theory of community food webs. III. Predicted and observed length of food chains. Proceedings of the Royal Society of London B 228: 317-353.

Cohen, J. E., F. Briand and C. M. Newman. 1990a. Community Food Webs: Data And Theory. Springer-Verlag, Berlin.

Cohen, J. E., T. Luczak, C. M. Newman and Z.-M. Zhou. 1990b. Stochastic structure and nonlinear dynamics of food webs: qualitative stability in a Lotka-Volterra cascade model. Proceedings of the Royal Society of London B 240: 607-627.

Cohen J. E. and C. M. Newman. 1984. The stability of large random matrices and their products. Annals of Probability 12: 283-310.

Cohen, J. E. and C. M. Newman. 1985a. When will a large complex system be stable? J. theoret. Biol. 113: 153-156.

Cohen, J. E. and C. M. Newman. 1985b. A stochastic theory of community food webs. I. Models and aggregated data. Proceedings of the Royal Society of London B 224: 421-448.

Cohen, J. E. and C. M. Newman. 1988. Dynamic basis of food web organization. Ecology 69: 1655-1664.

DeAngelis, A. L. 1975. Stability and connectance in food web models. Ecology 56: 238-243.

Gardner, M. R., and W. R. Ashby. 1970. Connectance of large, dynamical (cybernetic) systems: critical value for stability. Nature 228: 784 .

Gloss, G. P. and P. S. Lake. 1994. Spatial and temporal variation in the structure of an intermittent-stream food web. Ecol. Monog. 64: 1-21.

Hall, S. J. and D. G. Raffaelli. 1993. Food webs: theory and reality. Adv. Ecol. Res. 24: 187-239.

Havens, K. E. 1992. Scale and structure in natural food webs. Science 257: 1107-1109.

Havens, K. E. 1997. Unique structural properties of pelagic food webs. Oikos 78: 75-80.

Krebs, J. R. and N. B. Davies. 1993. An Introduction to Behavioural Ecology. $3^{\text {rd }}$ ed. Blackwell Science Ltd, Oxford.

Lawlor, L. R. 1978. A comment on randomly constructed ecosystem models. Amer. Nat. 112: 445-447.

Lawton, J. H. 1989. Food webs. In: J. M. Cherrett (ed.), Ecological Concepts: The Contribution of Ecology to an Understanding of the Natural World. Blackwell Scientific, Oxford, UK. pp. 4378

Lawton, J. H. 1992. Feeble links in food webs. Nature 355: 19-20.

Logofet, D. O. 1993. Matrices and Graphs-Stability Problems in Mathematical Ecology. CRC Press, Boca Raton. 
MacArthur, J. W. 1976. Environmental fluctuations and species diversity. In: M. Cody and J. M. Diamond (eds.), Ecology of species and communities. Harvard University Press, Cambridge, USA. pp. 74-80.

Martinez, N. D. 1991. Artifacts or attributes? Effects of resolution on the Little Rock Lake food web. Ecol. Monog. 61: 367-392.

Martinez, N. D. 1992. Constant connectance in community food webs. Amer. Nat. 139: 1208-1218.

Martinez, N. D., B. A. Hawkins, H. A. Dawah and B. P. Feifarek 1999. Effects of sampling effort on characterization of food web structure. Ecology 80: 1044-1055.

May, R. M. 1972. Will a large complex system be stable? Nature 238: 413-414.

May, R. M. 1974. How many species? Some mathematical aspects of the dynamics of populations. In: J. D. Cowan (ed.), Some Mathematical Problems in Biology. American Mathematical Society, Providence, USA. pp. 64-98.

Paine, R. T. 1980. Food webs: linkage, interaction strength and community infrastructure. J. Animal Ecol. 49: 667-685.

Paine, R. T. 1988. On food webs: road maps of interactions or the grist for theoretical development? Ecology 69: 1648-1654.

Paine, R. T. 1992. Food-web analysis through field measurements of per capita interaction strength. Nature 355: 73-75.

Pimm, S. L. 1980. Bounds on food web connectance. Nature 284: 591.

Pimm, S. L. 1982. Food Webs. Chapman and Hall, London, UK.
Pimm, S. L. 1984. The complexity and stability of ecosystems. $\mathrm{Na}$ ture 307: 321-326.

Polis, G. A. 1991. Complex trophic interactions in deserts: an empirical critique of food web theory. Amer. Nat. 138: 123-155.

Price, P. W. 1984. Insect Ecology. $2^{\text {nd }}$ Edition. John Wiley \& Sons. New York.

Rejmanek M. and P. Stary. 1979. Connectance in real biotic communities and critical values for stability of model ecosystems. $\mathrm{Na}$ ture 280: 311-313.

Schoener, T. W. 1989. Food webs from the small to the large. Ecology 70: 1559-1589.

Schoenly, K., R. A. Beaver and T. A. Heumier. 1991. On the trophic relations of insects: a food web approach. Amer. Nat. 137: 597638.

Schoenly, K. and J. E. Cohen. 1991. Temporal variation in food web structure: 16 empirical cases. Ecol. Monog. 61: 267-298.

Warren, P. H. 1990. Variation in food web structure: the determinants of connectance. Amer. Nat. 136: 689-700.

Warren, P. H. 1994. Making connections in food webs. Trends in Ecology and Evolution 9: 136-141.

Williams R. J. and N. D. Martinez. 2000. Simple rules yield complex food webs. Nature 404: 180-183.

Winemiller, K. O. 1989. Must connectance decrease with species richness? Amer. Nat. 134: 960-968. 